\title{
Évolution des temps de voyage et des vitesses en Suisse
}

Shipment breaks and commercial speed in Switzerland (1850-2010)

\section{Daniel Emery}

\section{CpenEdition}

\section{Journals}

Édition électronique

URL : https://journals.openedition.org/rhcf/1552

DOI : 10.4000/rhcf.1552

\section{Éditeur}

Rails \& histoire

\section{Édition imprimée}

Date de publication : 1 septembre 2012

Pagination : 191-199

ISSN : 0996-9403

\section{Référence électronique}

Daniel Emery, «Évolution des temps de voyage et des vitesses en Suisse », Revue d'histoire des

chemins de fer [En ligne], 42-43 | 2012, mis en ligne le 14 novembre 2014, consulté le 22 avril 2022 URL : http://journals.openedition.org/rhcf/1552 ; DOI : https://doi.org/10.4000/rhcf.1552 
Daniel EMERY

\section{Évolution des temps de voyage et des vitesses en Suisse}

\footnotetext{
e temps du voyage est souvent plus important pour le client du rail que la vitesse pure. En effet, l'augmentation de la vitesse ne réduit pas forcément le temps du voyage. Ce paradoxe s'explique aisément. Si, par exemple, la vitesse augmente de $20 \%$ mais que le tracé s'allonge de $30 \%$, le temps du voyage augmente de $8 \%$.
}

Le but de cet article est de montrer trois étapes associant le temps du voyage et la vitesse ferroviaire.

Avant-hier, la réduction du temps du voyage a été obtenue par la vitesse intrinsèque du chemin de fer naissant et cela malgré l'allongement du chemin parcouru.

Hier, et aujourd'hui encore, la réduction du temps de voyage ferroviaire est obtenue par l'arrivée de la grande vitesse sur des nouveaux tracés s'approchant de la ligne droite.

Aujourd'hui, et demain sûrement, la réduction du temps de voyage ferroviaire ne s'obtiendra plus tant par l'augmentation de la vitesse que par un horaire plus dense accompagné, du moins dans sa phase de développement, par un système idoine de correspondances. 
Tous les pays ne suivant pas cette démarche au même rythme, le présent article puise ses exemples dans un petit réseau ferré, fortement maillé et à relativement haute densité de population.

\section{Histoire}

La première ligne de chemins de fer en Suisse est la ligne StrasbourgBâle dont le premier terminus est en banlieue bâloise, à Saint-Jacques. Une première rupture de charge est supprimée lors du prolongement de la ligne et la construction d'une nouvelle gare intra-muros. La première ligne intérieure suisse n'est construite qu'en 1847. Ainsi, en 1850, la Suisse compte moins de $30 \mathrm{~km}$ de lignes ferrées, alors que la France a déjà à disposition un réseau plus grand que le réseau suisse à écartement UIC actuel.

Lorsque Stephenson, fils du célèbre constructeur de locomotives, est mandaté pour imaginer le futur réseau national, les ruptures de charges sont loin de ses préoccupations. La déclivité fait peur au chemin de fer naissant, même si l'architecte Carl Ritter von Ghega fait œuvre de pionnier sur les déclivités de $25 \%$ du Semmering autrichien. Toutefois, seules des locomotives disposant d'au moins quatre essieux moteurs couplés s'y aventurent à l'origine. Or, en 1850, cette technologie ne court pas les voies. Les larges vallées sont recherchées et toute rencontre avec un lac d'une certaine importance - aux rives escarpées - invite à abandonner le train pour le bateau pour franchir cette difficulté. Il est emblématique que la Compagnie naissante du Saint-Gothard arbore, à la fin du XIX ${ }^{e}$ siècle encore, un logo combinant la roue ferrée ailée, symbole de vitesse, au gouvernail d'un bateau des lacs Majeur et de Lugano.

L'autre peur, aussi bien technologique que financière, est la construction de longs tunnels. Pour le franchissement des reliefs jurassiens, Stephenson envisage un système de pousse funiculaire. Finalement, un tunnel de $2,5 \mathrm{~km}$ sera construit au Hauenstein avec l'aide de nombreuses cheminées d'accès. Cette technique, impraticable pour le massif alpin, pousse Stephenson à choisir le plus court des tunnels possibles au Lukmanier.

Grâce à l'influence prépondérante des cantons, le projet Stephenson a été considérablement adapté, ce qui a permis de ne pas laisser à l'écart des villes importantes et d'éviter des allongements de parcours évitables (fig. 1). 


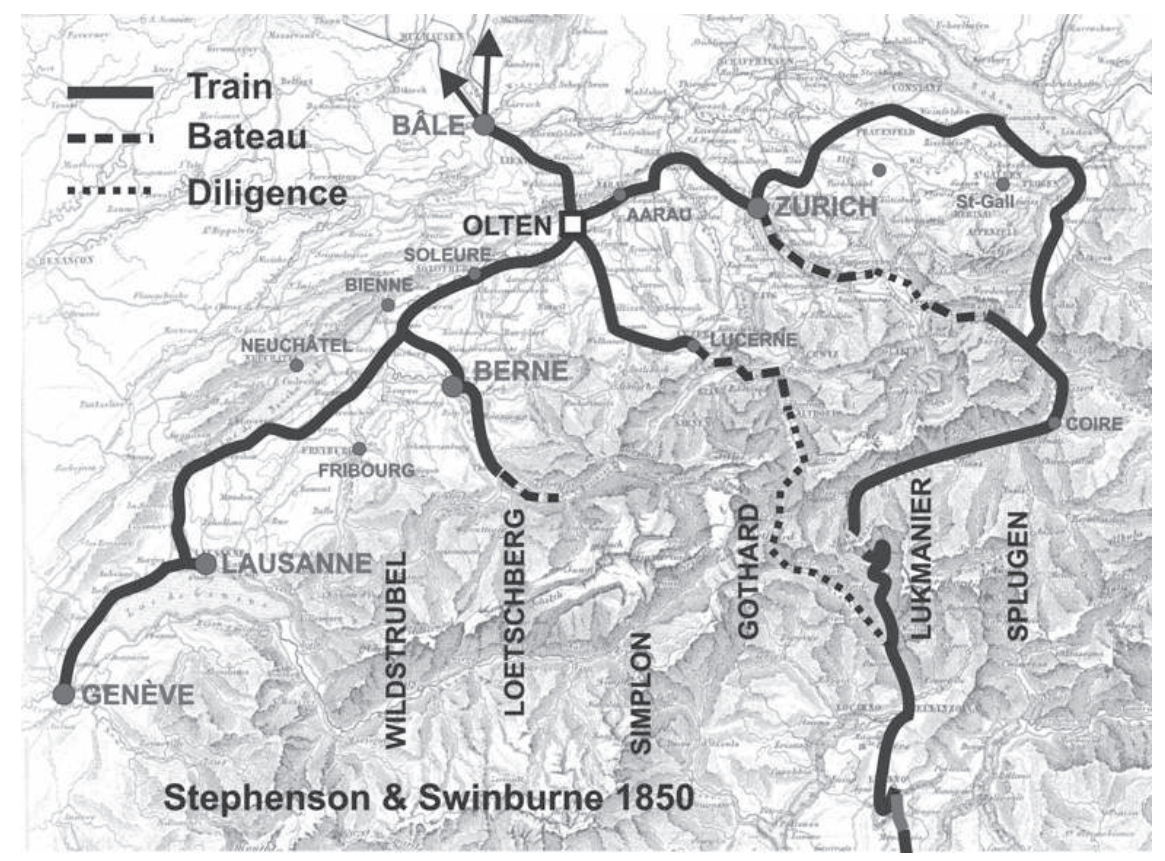

Figure 1. Proposition de réseau ferré suisse par Stephenson et Swinburne (1850). ${ }^{\odot}$ D. Emery.

\section{Les ruptures de charges intermodales pénalisent la vitesse commerciale}

Alors que la distance Bâle-Milan à vol d'oiseau est de $260 \mathrm{~km}$ environ, Stephenson prévoit un tracé deux fois plus long en s'écartant de la ligne directe de plus de $100 \mathrm{~km}$. La rupture de charge intermodale est fort pénalisante du point de vue de la vitesse, non pas en tant que telle (temps nécessaire pour le transbordement), mais à cause de la lenteur extrême des modes concurrencés par le chemin de fer. Ainsi, malgré des détours considérables, la vitesse fait un bond remarquable (tabl. 1). 
Tableau 1. Temps de parcours et vitesses sur l'axe Bâle - Milan ( 265 km en ligne droite), 1881-1883.

\begin{tabular}{l|c|c|c} 
& $\mathbf{1 8 8 1}$ - Saint-Gothard & Projet Stephenson-Lukmanier & 1883 - Saint-Gothard \\
\hline Moyen(s) de transport & Train $(96+41 \mathrm{~km})$ & & \\
\hline Bateau (40 km) & & & \\
\hline Diligence (184 km) & Train $\sim 515 \mathrm{~km})$ & Train $(379 \mathrm{~km})$ & \\
\hline Nombre de ruptures de charge & 5 & 0 & $379 \mathrm{~km}$ \\
\hline Longueur & $\sim 361 \mathrm{~km}$ & $\sim 515 \mathrm{~km}$ & \\
\hline Temps de parcours & $38 \mathrm{~h} 40 \mathrm{~min}$ & & \\
\hline (2 jours avec arrêt pour la nuit) & $\sim 17 \mathrm{~h}$ & & \\
\hline (grosse journée) & $12 \mathrm{~h} 16 \mathrm{~min}$ & & \\
\hline (soirée+nuit) & & & \\
\hline Vitesse commerciale & $\sim 9 \mathrm{~km} / \mathrm{h}$ & $\sim 30 \mathrm{~km} / \mathrm{h}$ & \\
\hline Vitesse euclidienne & $\sim 7 \mathrm{~km} / \mathrm{h}$ & $\sim 16 \mathrm{~km} / \mathrm{h}$ & $22 \mathrm{~km} / \mathrm{h}$ \\
\hline
\end{tabular}

Un constat important, tiré de l'exemple de l'axe Bâle-Milan, est que la vitesse commerciale n'est pas suffisante pour qualifier la rapidité du trajet. En effet, avec la même vitesse commerciale de $30 \mathrm{~km} / \mathrm{h}$, le temps de parcours Bâle-Milan via le Gothard en 1883 était de 12 h 16, alors qu'il aurait été de $40 \%$ supérieur via le Lukmanier. Il faut donc faire appel à la vitesse euclidienne (fig. 2). Cette mesure absolue de la vitesse permet de montrer que la réduction des temps de parcours dépend certes de l'augmentation de la vitesse, mais aussi du raccourcissement de la longueur du parcours. Savons-nous que la liaison ferroviaire Genève-Bordeaux passe par la LGV Sud-Est, Paris et la LGV Atlantique, et que sa vitesse euclidienne n'est que de $55 \mathrm{~km} / \mathrm{h}$, laissant ainsi de beaux jours aux compagnies aériennes?

Rappelons-nous que la vitesse commerciale entre Paris et Lyon n'a pas doublé avec l'arrivée des TGV, alors que la vitesse euclidienne, inversement proportionnelle au temps de parcours a, pour sa part, plus que doublé (tabl. 2). Il est peut-être aussi utile de mentionner l'écart important entre la vitesse plafond de $300 \mathrm{~km} / \mathrm{h}$ sur ligne à grande vitesse et les vitesses commerciale et euclidienne de l'ordre de $200 \mathrm{~km} / \mathrm{h}$, écart dû aux marges de régularité introduites, aux mises en vitesse et aux freinages, aux trajets terminaux sur lignes classiques, ainsi qu'aux $30 \mathrm{~km} / \mathrm{h}$, voire moins encore, imposés aux trains dans la gare en impasse de Paris-Lyon et dans la zone des quais avant arrêt dans les autres gares.

Tableau 2. Temps de parcours et vitesses sur l'axe Paris - Lyon (390 km en ligne droite), 1985-2010.

\begin{tabular}{l|c|c} 
& Via Dijon (1985) & Via la LGV (2010) \\
\hline Longueur & $511 \mathrm{~km} \mathrm{(=IABvol} \mathrm{+31 \% )}$ & $426 \mathrm{~km} \mathrm{(=IABvol} \mathrm{+9 \% )}$ \\
\hline Temps de parcours & $4 \mathrm{~h} 49$ & $1 \mathrm{~h} 56$ \\
\hline Vitesse commerciale & $118 \mathrm{~km} / \mathrm{h}(-)$ & $220 \mathrm{~km} / \mathrm{h}(+86 \%)$ \\
\hline Vitesse euclidienne & $90 \mathrm{~km} / \mathrm{h}(-)$ & $201 \mathrm{~km} / \mathrm{h}(+123 \%)$ \\
\hline
\end{tabular}


Figure 2. Vitesse commerciale et vitesse euclidienne.

\begin{tabular}{|c|c|}
\hline $\begin{array}{l}V_{\text {comABviac }}=\mathrm{l}_{\mathrm{ABviac}} / \mathrm{t}_{\mathrm{ABviaC}} \\
\mathrm{V}_{\text {euclABviaC }}=\mathrm{I}_{\mathrm{ABvol}} / \mathrm{t}_{\mathrm{ABviaC}}\end{array}$ & $\begin{array}{l}\text { peu importe le chemin emprunté } \\
\text { avec ses éventuels arrêts et } \\
\text { correspondances. }\end{array}$ \\
\hline $\begin{array}{l}V_{\text {comABviad }}=l_{A B v i a D} / t_{A B v i a D} \\
V_{\text {euclABviaD }}=I_{A B v o l} / t_{A B v i a D} \\
V_{\text {dispAB }[\mathrm{H} 1: \mathrm{H} 2]}=I_{A B v o l} / t_{A B m o y+}\end{array}$ & $\begin{array}{l}\text { La vitesse disponible (Vdisp) est } \\
\text { obtenue en divisant la distance } \\
\text { en ligne droite entre l'origine et } \\
\text { la destination par le temps moyen } \\
\text { séparant la prise de décision } \\
\text { de quitter l'origine pour se }\end{array}$ \\
\hline $\begin{array}{l}\text { La vitesse commerciale (Vcom) est } \\
\text { obtenue en divisant la longueur du } \\
\text { tracé par le temps séparant l'heure } \\
\text { de départ de l'origine et l'heure } \\
\text { d'arrivée à destination. Elle est } \\
\text { indépendante des éventuels arrêts } \\
\text { et correspondances mais dépend } \\
\text { du tracé. }\end{array}$ & $\begin{array}{l}\text { à destination. En I'absence de } \\
\text { pondération, il est supposé que } \\
\text { cette décision est prise aléatoire- } \\
\text { ment dans l'intervalle temporel } \\
\text { allant de I'heure H1 à I'heure } \\
\text { Hn (disponibilité temporelle). } \\
\text { Pour le chemin de fer, la vitesse } \\
\text { disponible dépend de l'horaire } \\
\text { et du maillage du réseau, mais } \\
\text { ne dépend plus du tracé. Cette }\end{array}$ \\
\hline $\begin{array}{l}\text { La vitesse euclidienne (Veucl) est } \\
\text { obtenue en divisant la distance } \\
\text { en ligne droite entre l'origine et la } \\
\text { destination par le temps séparant } \\
\text { I'heure de départ de l'origine et } \\
\text { I'heure d'arrivée à destination, }\end{array}$ & $\begin{array}{l}\text { notion de disponibilité permet } \\
\text { l'établissement d'une relation } \\
\text { univoque entre la vitesse et le } \\
\text { temps de parcours, attente de } \\
\text { I'heure de départ comprise. }\end{array}$ \\
\hline
\end{tabular}

\section{Les courbes pénalisent la vitesse commerciale}

Prévue originellement pour une vitesse de $100 \mathrm{~km} / \mathrm{h}$, la ligne du SaintGothard va devoir accepter, pour des raisons financières, des courbes ayant des rayons de $300 \mathrm{~m}$ seulement. Cela correspond, selon la législation suisse, a une de vitesse maximum de $80 \mathrm{~km} / \mathrm{h}$ pour des trains sans inclinaison de caisses. Dès la seconde partie du $\mathrm{xx}^{\mathrm{e}}$ siècle, cette vitesse peut être tenue en permanence à la montée grâce aux puissantes locomotives électriques. Elle peut aussi, quelque temps après, être tenue à la descente grâce au freinage de retenue électrique remplaçant le freinage pneumatique dont l'usage occasionnait une variation de la vitesse en dents de scie.

Sur les lignes du Plateau suisse, aux tracés en plan à peine plus favorables, les vitesses commerciales restent fort modestes, à l'exemple de la ligne Lausanne-Berne dont la vitesse commerciale oscille entre 80 et $90 \mathrm{~km} / \mathrm{h}$ depuis plus de cinquante ans, au gré de la politique d'arrêts. 
Pour augmenter encore la vitesse sur les parcours sinueux sans péjorer le confort des voyageurs, il est certes fait appel à des techniques d'inclinaison plus ou moins prononcées des caisses, mais les gains obtenus en vitesse commerciale sont considérablement inférieurs à ce que pourrait permettre la construction de nouveaux tronçons ferroviaires plus rectilignes.

\section{Les ruptures de charges intramodales ne pénalisent que très peu la vitesse commerciale}

Au début du $\mathrm{xx}^{\mathrm{e}}$ siècle des dizaines de chemins de fer secondaires sont construits en écartement métrique. Ainsi, en 1983, lors de la mise en place de l'horaire cadencé, les lignes à voie étroite, gérées par une vingtaine de compagnies différentes, assurent les correspondances avec le réseau à voie normale dans plus de quarante gares d'échange où la rupture de charge est imposée pour des raisons techniques depuis le début du $\mathrm{Xx}^{\mathrm{e}}$ siècle. Dans la plupart de ces gares d'échange, la correspondance ne prend que quelques minutes (tabl. 3).

Tableau 3. Exemple de temps nécessaire pour la correspondance en 2010 sur le réseau ferré suisse.

\begin{tabular}{l|c|c|c} 
Gare & Compagnie sur voie normale & Compagnie sur voie métrique & Temps correspondance \\
\hline Nyon & Chemins de Fer Fédéraux suisses & Nyon-St.Cergue-Moret (NStCM) & 3 min \\
\hline Coire & Chemins de Fer Fédéraux suisses & Rhätische Bahn (RhB) & 4 min \\
\hline Interlaken & Bern-Lötschberg-Simplon (BLS) & Zentral Bahn (ZB) & 5 min \\
\hline Herisau & Südostbahn (SOB) & Appenzeller Bahnen (AB) & $3 \min$ \\
\hline
\end{tabular}

Ainsi, à un temps de parcours domicile-travail la correspondance n'ajoute souvent que moins de $10 \%$. De plus, même sur le réseau fortement maillé des CFF il serait économiquement impossible d'aller de partout à partout sans changer de train, même en renforçant considérablement la capacité des lignes formant ce réseau. Les Suisses sont donc habitués depuis fort longtemps à des changements rapides de trains, parfois avec skis ou vélos.

Alors que la France réalise sa première ligne à grande vitesse (LGV), les CFF envisagent aussi la construction de nouvelles infrastructures permettant une augmentation significative des vitesses: les Nouvelles Transversales Ferroviaires (NTF). Cette tentative se solde par un échec car les régions périphériques se sentent délaissées. Les CFF ne conserveront qu'un court nouveau tronçon à $200 \mathrm{~km} / \mathrm{h}$ de cet ancien projet et proposent alors le projet Rail 2000 basé sur les correspondances. Ils le vendent au peuple suisse, non pas grâce 
à la croissance de la vitesse, mais grâce à la matrice des temps de parcours. Ils prennent grand soin de montrer les gains de temps de parcours obtenus par les régions périphériques. Ce gain n'est certes pas obtenu sur le territoire des régions décentralisées, mais ces dernières en bénéficient grâce aux bonnes correspondances : c'est l'avènement de l'horaire cadencé coordonné. Le vocabulaire change en conséquence? il n'est plus fait mention de rupture de charge mais de nœud de correspondance.

\section{Certaines ruptures de charges augmentent la vitesse disponible moyenne}

La vitesse commerciale disponible moyenne et la vitesse euclidienne disponible moyenne permettent d'intégrer la disponibilité temporelle de l'offre ferroviaire. La vitesse disponible moyenne prend en compte non seulement le temps de parcours mais aussi le temps moyen d'attente avant de commencer le parcours. Sous sa forme la plus simple, la vitesse disponible moyenne considère une arrivée équiprobable des voyageurs sur toute la période considérée. Cette nouvelle notion permet de comparer le mode ferroviaire, lorsqu'il est basé sur un horaire relativement dense, et le mode routier individuel à disponibilité immédiate.

Ainsi, bien que la vitesse commerciale soit souvent restée constante ces cinquante dernières années, l'offre s'est considérablement étoffée. Or, à partir d'une fréquence à la demi-heure, l'horaire perd de son importance. Le professionnel en séance de travail dans une autre ville ne va plus avoir les yeux rivés sur sa montre, écourtant parfois son entrevue. Il sait que, même s'il joue de malchance, son attente ne dépassera pas une demi-heure, attente qu'il pourra supporter grâce à l'expresso et à la liaison WiFi disponibles en gare. Entre Berne et Lausanne, la vitesse euclidienne disponible moyenne est passée de $41 \mathrm{~km} / \mathrm{h}$ en 1960 à $55 \mathrm{~km} / \mathrm{h}$ en 2000 . Cela devient suffisant pour renoncer à conduire sa voiture (vitesse disponible d'environ $65 \mathrm{~km} / \mathrm{h}$ de centre à centre), en particulier si une partie du voyage en train peut être consacrée à des tâches professionnelles.

Le cas actuel Lausanne-Bâle est significatif des gains en vitesse disponible moyenne (tabl. 4). 
Tableau 4. Temps de parcours et vitesses sur I'axe Lausanne - Bâle (135 km en ligne droite), 2010.

\begin{tabular}{l|c|c|c} 
& $\begin{array}{c}\text { Via.Bienne (sans } \\
\text { transbordement à } \\
\text { Bienne) }\end{array}$ & $\begin{array}{c}\text { Via Bienne (avec ou sans } \\
\text { transbordement à } \\
\text { Bienne) }\end{array}$ & $\begin{array}{c}\text { Via Bienne ou Berne (avec } \\
\text { ou sans transbordement } \\
\text { dans l'une de ces gares) }\end{array}$ \\
\hline Fréquence & toutes les 2 heures & horaire & semi-horaire \\
\hline Nombre de trains (6 h - 20 h) & 8 trains & 15 trains & 29 trains \\
\hline Temps de parcours & $2 \mathrm{~h} 08 \mathrm{~min}$ & $2 \mathrm{~h} 08 \mathrm{~min}$ & $2 \mathrm{~h} 08 \mathrm{~min}$ ou $2 \mathrm{~h} 10 \mathrm{~min}$ \\
\hline Longueur & $179 \mathrm{~km}$ & $179 \mathrm{~km}$ & $179 \mathrm{~km}$ ou $198 \mathrm{~km}$ \\
\hline Vitesse commerciale & $84 \mathrm{~km} / \mathrm{h}$ & $84 \mathrm{~km} / \mathrm{h}$ & $84 \mathrm{~km} / \mathrm{h}$ ou $91 \mathrm{~km} / \mathrm{h}$ \\
\hline Vitesse euclidienne & $63 \mathrm{~km} / \mathrm{h}$ & $63 \mathrm{~km} / \mathrm{h}$ & $63 \mathrm{~km} / \mathrm{h}$ ou $62 \mathrm{~km} / \mathrm{h}$ \\
\hline Vitesse disponible moyenne & $43 \mathrm{~km} / \mathrm{h}$ & $51 \mathrm{~km} / \mathrm{h}$ & $56 \mathrm{~km} / \mathrm{h}$ \\
\hline
\end{tabular}

Une telle mise en réseau de l'offre impose de discipliner les trains dans les sillons offrant les correspondances. La disponibilité temporelle du train, augmentant avec l'augmentation des fréquences d'une part et les possibilités de correspondances d'autre part, impose une banalisation des sillons. Ce ne sont plus les correspondances qui s'adaptent aux trains les plus rapides, mais les temps de parcours des trains les plus rapides qui sont domestiqués afin de s’intégrer dans le système de correspondance.

\section{Une offre généralisée au quart d'heure augmentera la vitesse disponible et rendra obsolète le système de correspondances}

Peu à peu le réseau principal suisse est exploité par des trains circulant à la cadence semi-horaire. Cette fréquence n'est toutefois pas encore assez élevée pour pouvoir renoncer à assurer des correspondances spécifiques entre les trains. Cependant, déjà pour le trafic RER, la fréquence au quart d'heure progresse continuellement. Sur le tronçon national phare, entre Berne et Zürich, l'offre de trains sans arrêt à la demi-heure n'est plus suffisante pour assurer à la clientèle un excellent confort de transport aux heures de pointe malgré l'utilisation d'un matériel à deux étages.

La mise en place d'une offre au quart d'heure atténue considérablement l'écart entre la vitesse euclidienne et la vitesse disponible. Par exemple, entre Lausanne et Bâle, la vitesse disponible serait de $59 \mathrm{~km} / \mathrm{h}$ pour une offre au quart d'heure, soit de seulement $4 \mathrm{~km} / \mathrm{h}$ inférieure à la vitesse commerciale (tableau 4). Avec une offre généralisée au quart d'heure, il devient possible de renoncer à la coordination des horaires des lignes, les correspondances étant basées uniquement sur la densité de cette offre, proche de celle d'un métro. 
Toutefois, les grands nœuds de correspondances, déjà largement congestionnés au moins deux fois par heure, rendent quasiment impossible le passage systématique à la fréquence au quart d'heure sans construction d'un nouvel étage aux gares existantes (voir les projets actuels à Zürich, Berne, Lucerne, Lausanne...) et de quelques sauts-de-mouton.

\section{Conclusion}

La mise en place d'un réseau ferré a permis de raccourcir considérablement le nombre de jours de voyage pour parcourir quelques centaines de kilomètres. Puis la vitesse de plus en plus élevée des trains a permis d'effectuer un aller-retour dans la journée entre des agglomérations distantes de quelques centaines de kilomètres tout en ayant à disposition une demi-journée à destination. L'arrivée de la très grande vitesse ferroviaire a permis d'étendre cette possibilité à des agglomérations plus éloignées les unes des autres et d'offrir une pleine journée de travail à destination pour de nombreuses agglomérations directement connectées au réseau à grande vitesse.

Toutefois, aussi bien la vitesse de croisière que la vitesse commerciale ferroviaire ne peuvent donner une bonne base de comparaison avec l'alternative routière car ces vitesses sont trop dépendantes du tracé, d'une part, et des temps d'attente pour commencer son trajet en train, d'autre part.

La vitesse disponible est, en revanche, un indicateur pertinent pour qualifier objectivement la performance de l'offre ferroviaire entre deux lieux. Elle permet une comparaison simple et directe avec l'offre routière, grâce à la prise en compte de la couverture temporelle de l'offre ferroviaire. L'horaire cadencé à l'heure est une première étape vers une couverture temporelle permettant de concurrencer l'usage de la voiture privée.

La mise en place d'une offre au quart d'heure sur un réseau fortement maillé peut être considérée comme un véritable passage à une exploitation de type métro, les «stations de correspondance " assurant leur fonction sans que les horaires des lignes aient besoin d'être coordonnés.

Ainsi, le prestige de la vitesse sur des tronçons bien déterminés cèdera le pas à une performance d'ensemble permettant de se déplacer de partout à partout, rapidement et en tout temps. 
